\title{
Region based Structure Preserving Nonnegative Matrix Factorization for Hyperspectral Unmixing
}

\author{
Lei Tong, Jun Zhou, Senior Member, IEEE, Xue Li, Yuntao Qian, Member, IEEE and Yongsheng Gao, Senior \\ Member, IEEE
}

\begin{abstract}
Hyperspectral unmixing is one of the most important techniques in remote sensing image analysis. In recent years, nonnegative matrix factorization (NMF) method is widely used in hyperspectral unmixing. In order to solve the nonconvex problem of NMF method, a number of constraints have been introduced into NMF models, including sparsity, manifold, smoothness, et al. However, these constraints ignore an important property of hyperspectral image, i.e., the spectral responses in a homogeneous region are similar at each pixel but vary in different homogeneous regions. In this paper. We introduce a novel region based structure preserving NMF (R-NMF) to explore consistent data distribution in the same region while discriminating different data structures across regions in the unmixed data. In this method, a graph cut algorithm is first applied to segment the hyperspectral image to small homogeneous regions. Then two constraints are applied to the unmixing model, which preserve the structural consistency within region while discriminating the differences between regions. Results on both synthetic and real data have validated the effectiveness of this method, and shown that it has outperformed several state-of-theart unmixing approaches.
\end{abstract}

Index Terms-Hyperspectral Unmixing, Nonnegative Matrix Factorization, Homogeneous Region.

\section{INTRODUCTION}

With rapid development of imaging instruments, hyperspectral imagery has been widely used in environmental monitoring, agriculture, and mining [1], [2], [3], [4]. Pixels in remote sensing hyperspectral imagery are normally composed of mixed spectral responses from multiple ground objects due to the low spatial resolution of the data. Therefore, hyperspectral unmixing has become an important technique to decompose mixed pixels into a collection of spectral signatures, i.e., endmembers, and their corresponding proportions, i.e., abundances [5], [6].

Many unmixing methods are based on the linear mixing model which treats each pixel as a linear combination of endmembers and assume there is no interference between them [7], [8]. These methods can be divided into three main categories: geometry based, sparse regression based, and statistics based.

L. Tong and Y. Gao are with School of Engineering, Griffith University, Nathan, Australia.

J. Zhou is with School of Information and Communication Technology, Griffith University, Nathan, Australia. Corresponding author: J. Zhou (jun.zhou@griffith.edu.au)

$\mathrm{X}$. Li is with School of Computer Science and Engineering, Nanjing University of Science and Technology, Nanjing, China

Y. Qian is with the Institute of Artificial Intelligence, College of Computer Science, Zhejiang University, Hangzhou, China.
Geometry based methods explore the geometric relationship between candidate endmembers and often treat them in a projected space. Classic methods in this category include $\mathrm{N}$ FINDR [9], pixel purity index (PPI) [10], and vertex component analysis (VCA) [11]. The drawback of these methods is that they assume pure pixels present in the image which is not always the case. On the contrary, simplex identification via split augmented Lagrangian (SISAL) method [12] does not have this assumption. It enforces the endmembers to form a convex hull containing all pixels in the image data, and then estimate the endmembers and abundance using a sequence of augmented Lagrangian optimizations.

Sparse regression methods reconstruct the hyperspectral image as a sparse linear combination of few endmembers out of a pool of candidates [13], [14]. The sparsity constraint can be applied to the spectral dimension so that only those with high variances of material reflectance need to be selected [15]. It also can deal with the problem when the signatures in the library and in the real data are mismatched [16]. On top of the modelling in the spectral domain, spatial regularization term can be incorporated into the sparse model, for example, via total variation [17], or spectral constraint with regularization term defined based on prior knowledge of endmembers [18].

Statistical unmixing methods include independent components analysis (ICA) [19], [20], normal compositional model [21] and nonnegative matrix factorization (NMF) [22]. NMF decomposes image data into nonnegative endmember and abundance matrices. However, NMF is a non-convex problem which does not have unique solution and is strongly influenced by the initialization step. In order to overcome this drawback, various constraints have been introduced to regularize NMF method according to different considerations on the property of hyperspectral image. Wang and Du [23] set signature dissimilarity as the constraint on endmembers. Liu et al [24] proposed that weights can be assigned to local neighborhood to enhance the unmixing performance. Sparsity has also been used as a spatial constraint in NMF method, which allows each pixel be decomposed into one or few endmembers [25], [26]. Some methods combine spectral and spatial constraints, for example, to force the smoothness of both endmembers and abundance [27], to introduce abundance separation and smoothness [28], to maintain the manifold structure of raw image and unmixed data [29], or to maintain structure information by clustering [30]. Recently, Yuan proposed an NMF method [31] which is similar to sparse regression method and needs a library of signatures. In this method, a projection-based nonnegative matrix factorization 
algorithm is implemented by importing spectra library into the NMF framework, with the endmember signatures being adaptively generated from the spectral library. Li et al [32], on the other hand, developed a robust collaborative NMF method to reduce the number of overestimated endmembers during the unmixing process. Prior knowledge has also been considered in NMF based method to enhance the unmixing performance by introducing known endmember into the model [33], [34].

While most unmixing methods are operated at pixel level, some researchers explored region based unmixing. David et al [35] proposed to segment a hyperspectral image into superpixels and then estimate the endmembers on each superpixel. The results show that region based operation has led to better performance than methods implemented on pixels. Gabriel and Antonio [36] proposed an unsupervised spatial preprocessing module which adopts a region selection approach using orthogonal projections. After region selection, an automatic endmember extraction approach is used to extract endmembers. Eches et al [37] proposed a Bayesian based unmixing method which incorporates spatial correlations between partitioned regions into consideration. These methods show that regions play an important role in the unmixing problem because they allow reduced influence from noises in hyperspectral images by exploring the spatial information of neighbouring pixels [35].

We assume there is strong semantic relationship of regions in a hyperspectral image. This is mainly due to the fact that the same land covers tend to appear in a local neighborhood, for example, buildings, roads, forests etc.. We define those regions mainly containing the same endmembers as homogeneous regions. Therefore it is reasonable to assume consistent abundances in these homogeneous regions, in particular when one or very few endmembers present at a pixel. On the other hand, the same endmembers may appear in many regions, for example, soils and trees may appear in many locations on an image and form mixed pixels with other endmembers. Their contribution to each region shall be distinctive, which forms the reason for discriminating the contribution from endmembers across regions.

Inspired by the advantages of exploring regional-wise data distribution, we propose a novel region based NMF (R-NMF) method for hyperspectral unmixing. This method aims at enforcing consistent abundances within homogeneous regions while discriminating the contribution from endmembers across regions. R-NMF consists of several steps. First, a graph cut algorithm is employed to segment a hyperspectral image into small homogeneous regions. Then two constraints are added to a sparse NMF model. The first constraint is to make the abundance within homogeneous regions be similar. The second one is a structure preserving constraint which defines the relationship between homogeneous regions so as to discriminate their differences. We adopt the k-nearest and the k-farthest neighbours to preserve the structural information between homogeneous regions.

The rest of this paper is organized as follows. Section II describes the background on linear mixture model and $L_{1 / 2^{-}}$ NMF method. Section III makes detailed description on the proposed method, including homogeneous region segmenta- tion approach and region based NMF. Section IV presents the experimental results on both synthetic and remote sensing data. Finally, conclusions are drawn in Section V.

\section{RELATED WORK ON NMF-BASED HYPERSPECTRAL UNMIXING}

In this section, we describe the basic linear mixture model for hyperspectral unmixing and its sparsity constrained extension $L_{1 / 2}$-NMF. These form the basis of the proposed method.

\section{A. Linear Mixture Model}

In linear mixture model, each pixel can be considered as the linear combination of several endmembers. Let the number of wavelength-indexed bands in an image be $H$ and the number of endmembers be $P$. A pixel $\mathbf{y}$ in a hyperspectral image $\mathbf{Y}$ is an $H \times 1$ column vector whose entries correspond to the reflectance of object in different bands. Let $\mathbf{M}$ be an $H \times P$ endmember matrix $\left(\mathbf{m}_{1}, \ldots, \mathbf{m}_{j}, \ldots, \mathbf{m}_{P}\right)$, where $\mathbf{m}_{j}$ is an $H \times 1$ column vector representing the spectral signature of the $j^{t h}$ endmember. Then $\mathbf{y}$ can be approximated by a linear combination of endmembers

$$
\mathbf{y}=\mathbf{M r}+\mathbf{e}
$$

where $\mathbf{r}$ is a $P \times 1$ column vector for endmember abundances, and $\mathbf{e}$ is the additive Gaussian white noise.

Let $N$ be the number of pixels in image $\mathbf{Y}$, the matrix form of the linear mixture model can be defined as

$$
\mathbf{Y}=\mathbf{M R}+\mathbf{E}
$$

where $\mathbf{R}$ is a $P \times N$ abundance matrix which contains the proportion of each endmember at each pixel. $\mathbf{E}$ is an $H \times N$ matrix representing the additive noise.

\section{B. $L_{1 / 2}$ Regularized NMF}

The goal of hyperspectral unmixing is to estimate the endmember matrix $\mathbf{M}$ and the abundance matrix $\mathbf{R}$ given a hyperspectral image $\mathbf{Y}$. Apparently, both $\mathbf{M}$ and $\mathbf{R}$ shall be nonnegative because spectral responses of endmembers and their proportion at each pixel can not be smaller than zero. Because of this property, nonnegative matrix factorization (NMF) [38], [39], which decomposes a matrix into nonnegative matrices, becomes a natural solution to hyperspectral unmixing. It estimates the endmember matrix $\mathbf{M}$ and abundance matrix $\mathbf{R}$ simultaneously to reconstruct the high dimensional data matrix $\mathbf{Y}$. To cope with the non-convexity of the original NMF method and model the sparsity of abundance, an $L_{1 / 2}$ regularizer has been introduced as a sparsity constraint which leads to $L_{1 / 2}$-NMF [25]. The objective function of $L_{1 / 2}$-NMF is

$$
\operatorname{obj}(\mathbf{M}, \mathbf{R})=\frac{1}{2}\|\mathbf{Y}-\mathbf{M R}\|_{F}^{2}+\lambda\|\mathbf{R}\|_{\frac{1}{2}}
$$

where $\|\cdot\|_{F}$ represents the Frobenius norm, and

$$
\|\mathbf{R}\|_{1 / 2}=\sum_{p=1}^{P} \sum_{n=1}^{N} \mathbf{R}_{p n}^{1 / 2}
$$


A solution for minimizing this object function is the multiplied iterative algorithm [39], [22]. When applied to Equation (3), the multiplicative rule is given by the following two equations:

$$
\begin{gathered}
\mathbf{M} \leftarrow \mathbf{M} . * \mathbf{Y} \mathbf{R}^{T} \cdot / \mathbf{M R} \mathbf{R}^{T} \\
\mathbf{R} \leftarrow \mathbf{R} \cdot * \mathbf{M}^{T} \mathbf{Y} \cdot /\left(\mathbf{M}^{T} \mathbf{M R}+\frac{\lambda}{2} \mathbf{R}^{-\frac{1}{2}}\right)
\end{gathered}
$$

where $(.)^{T}$ is the transpose of the matrix, .* and ./ denote element-wise multiplication and division, respectively.

\section{APPROACH}

In this section, we first introduce the homogeneous region segmentation method and then describe the proposed R-NMF method in detail. At last some implementation issues are discussed.

\section{A. Homogeneous Region Segmentation Approach}

The first step in our method is to segment hyperspectral image into a set of homogeneous regions. This is an unsupervised segmentation problem and can be implemented by an efficient graph-based method [40]. In this method, each pixel in a hyperspectral image can be represented as a vertex $v_{i} \in V, i=1, \ldots, N$. An edge $E\left(v_{i}, v_{j}\right)$ connects a vertex $v_{i}$ with its neighbour $v_{j}$, and the weight of the edge is related to the distance between the connected vertices.

An iterative algorithm segments the image to $Q$ segments $\left\{G_{1}, \ldots, G_{Q}\right\}$. At beginning of the iteration, each pixel is treated as a separate region. Then the algorithm merges the pixels to form homogeneous regions according to pairwise region comparison. Two criteria are used for the merging: the maximum internal difference (MID) of a region and the minimum connecting weight (MCW) between regions. The maximum internal difference is the largest weight in the minimum spanning tree $M S T(G)$ [41] of a homogeneous region $G$, which is defined as follows

$$
\begin{aligned}
M I D(G)=\max \omega\left(v_{i}, v_{j}\right)+\frac{\rho}{|G|} \quad & \forall v_{i} \in G, v_{j} \in G \\
& \left(v_{i}, v_{j}\right) \in \operatorname{MST}(G)
\end{aligned}
$$

where $\rho$ is a parameter that controls the contribution of small regions. To calculate the weight of an edge, the spectral angle distance (SAD) is used, such that

$$
\omega\left(v_{i}, v_{j}\right)=\arccos \left(\frac{\mathbf{y}_{i}^{T} \mathbf{y}_{j}}{\left\|\mathbf{y}_{i}\right\|\left\|\mathbf{y}_{j}\right\|}\right)
$$

The minimum connecting weight between homogeneous regions $G_{1}$ and $G_{2}$ can be calculated by

$$
\begin{array}{cl}
M C W\left(G_{1}, G_{2}\right)=\min \omega\left(v_{i}, v_{j}\right) \quad & \forall v_{i} \in G_{1}, v_{j} \in G_{2} \\
& \left(v_{i}, v_{j}\right) \in E
\end{array}
$$

With the calculated maximum internal difference and minimum connecting weight between homogeneous regions, whether two homogeneous regions should be merged or not can be determined as follows: if the maximum internal differences from both homogeneous regions are larger than the minimum connecting weight between two regions, these two regions shall be merged. Otherwise, they shall remain separated. The merging process can be iterated until no changes on the regions happen. Finally, a threshold $T$ is used to control the minimal size of the homogeneous region. Regions smaller than this threshold is merged to the nearest region. This is an effective way to reduce the influence of small regions created due to noises in the image.

\section{B. Region Based NMF}

In this subsection, we describe the region based NMF approach. It consists of two main process: structure consistency in homogeneous region and structure preservation between homogeneous regions. To describe this approach, we define $\mathbf{c}_{q}$ as the mean value of spectral responses of region $G_{q}$, and $\alpha_{q}$ as the representation in the space spanned by $\mathbf{c}_{q}$ of region $G_{q}$. Variable $\alpha_{q}$ can be considered as the mean abundance of the homogeneous region.

1) Structure Consistency in Homogeneous Region: After $Q$ homogeneous regions $G=\left\{G_{1}, \ldots, G_{Q}\right\}$ are generated, we calculate the mean value of spectral responses $c_{q}$ of each region $G_{q}$. For each homogeneous region, the spectral responses of pixels in the region shall be similar. Based on the manifold learning theory [42], the raw spectral responses can be treated as high dimensional data and the abundance is considered as the mapped low dimensional data. It means that in the homogeneous region, the abundance of each pixel should be similar to each other. Therefore, we set a constraint between the mean abundance $\alpha_{q}$ and the estimated abundance at each pixel $r_{n}$ of region $G_{q}$. The object function is defined as follows

$$
\begin{array}{r}
\operatorname{obj}(\mathbf{M}, \mathbf{R})=\frac{1}{2}\|\mathbf{Y}-\mathbf{M R}\|_{F}^{2}+\sum_{q=1}^{Q} \sum_{n \in G_{q}}\left\|\mathbf{r}_{n} \lambda_{q}^{2}\right\|_{1 / 2} \\
+\mu \sum_{q=1}^{Q} \sum_{n \in G_{q}}\left\|\mathbf{r}_{n}-\alpha_{q}\right\|_{2}^{2}
\end{array}
$$

where the second term controls the sparsity of abundance in each homogeneous region. $\lambda_{q}$ is a parameter that controls the contribution of the sparsity term, which is defined by Qian $e t$ al [25] as

$$
\lambda_{q}=\frac{1}{\sqrt{H}} \sum_{h} \frac{\sqrt{N}-\left\|r_{q h}\right\|_{1} /\left\|r_{q h}\right\|_{2}}{\sqrt{N-1}}
$$

where $r_{q h}$ denotes the $q$-th homogeneous region of the $h$-th band in the hyperspectral imagery. $H$ and $N$ are the total numbers of bands and pixels, respectively. $\mu$ is a parameter that controls the contribution from the structure consistency term in Equation (10). The larger $\mu$ is, the higher similarity of abundance in homogeneous regions.

To solve Equation (10), we rewrite the objective function in column form as follows

$$
\begin{aligned}
\operatorname{obj}\left(\mathbf{M}, \mathbf{r}_{q}(n)\right)= & \frac{1}{2}\left\|\mathbf{y}_{q}(n)-\mathbf{M r}_{q}(n)\right\|_{2}^{2}+\left\|\mathbf{r}_{q}(n) \lambda_{q}^{2}\right\|_{1 / 2} \\
& +\mu\left\|\mathbf{r}_{q}(n)-\alpha_{q}\right\|_{2}^{2}
\end{aligned}
$$


where $\mathbf{y}_{q}(n)$ and $\mathbf{r}_{q}(n)$ are the $n$-th spectral signature and corresponding estimated abundance vector in homogeneous region $G_{q}$, respectively. $\alpha_{q}$ is the mapped representation of $\mathbf{c}_{q}$ in the new P-dimension space. The first two terms are the same as $L_{1 / 2}$-NMF. The third term is the consistency term. It minimizes the difference between $\mathbf{r}_{q}(n)$ and $\alpha_{q}$, making the estimated abundance in the homogeneous region similar. Method to estimate $\alpha_{q}$ will be described in Section III-B2.

Please note that the estimation of $\mathbf{M}$ remains the same as the $L_{1 / 2}$-NMF solution via Equation (5), we only describe how $\mathbf{r}_{q}(n)$ should be updated. According to the gradient descent algorithm, $\mathbf{r}_{q}(n)$ can be updated as follows

$$
\begin{gathered}
\mathbf{r}_{q}(n) \leftarrow \mathbf{r}_{q}(n)-\eta\left(-\mathbf{M}^{T}\left(\mathbf{y}_{q}(n)-\mathbf{M r}_{q}(n)\right)+\right. \\
\left.\frac{\lambda_{q}}{2} \mathbf{r}_{q}(n)^{-\frac{1}{2}}+2 \mu\left(\mathbf{r}_{q}(n)-\alpha_{q}\right)\right)
\end{gathered}
$$

Let

$$
\eta=\frac{\mathbf{M}}{\mathbf{M}^{T} \mathbf{M} \mathbf{r}_{q}(n)+\frac{\lambda_{q}}{2} \mathbf{r}_{q}(n)^{-\frac{1}{2}}+2 \mu \mathbf{r}_{q}(n)}
$$

the above step becomes

$$
\begin{aligned}
\mathbf{r}_{q}(n) \leftarrow & \mathbf{r}_{q}(n) \cdot *\left(\mathbf{M}^{T} \mathbf{y}_{q}(n)+2 \mu \alpha_{q}\right) \cdot /\left(\mathbf{M}^{T} \mathbf{M} \mathbf{r}_{q}(n)\right. \\
& \left.+\frac{\lambda_{q}}{2} \mathbf{r}_{q}(n)^{-\frac{1}{2}}+2 \mu \mathbf{r}_{q}(n)\right)
\end{aligned}
$$

2) Structure Preservation between Homogeneous Regions: Structure preserving aims to properly preserve the local affinity of data distribution before and after matrix factorization and at the same time avoid the distortion caused by distant data points. The latter property is also called distant repulsion [43]. The effect of structured discrimination is illustrated in Fig. 1. Fig. 1(a) shows the distribution of two materials, Fig. 1(b) is an example of the distribution of estimated abundance when only local affinity property is considered. This reduces to the MRSNMF [29] method. It can be clearly seen that the local affinity may result in overlaps (data in the red rectangle) between different materials. Conversely, Fig. 1(c) indicates that only modelling the repulsion correlations without paying attention to the local affinity property leads to loose clusters, i.e., the data is highly dispersed. For homogeneous region generated from hyperspectral images, local affinity property means that the abundance of the same material in different regions should be similar. Meanwhile, distant repulsion property forces the abundance of different materials, such as grass and tree, be different.

In the proposed R-NMF method, graph regularization is used to preserve the structural information. The local affinity of graph vertices which represent the reflectance at different data points can be defined as follows

$$
\min _{\alpha} \frac{1}{2} \sum_{i=1}^{Q} \sum_{j=1}^{Q} \mathbf{W}_{i j}^{n}\left\|\alpha_{i}-\alpha_{j}\right\|^{2}
$$

where $\mathbf{W}^{n}$ is a weight matrix constructed from the pairwise distance between two regions $G_{i}$ and $G_{j}$. In reality, this can be simplified by only considering the $k$ nearest neighbours of $\alpha_{i}$ instead of the whole dataset. The entries of $\mathbf{W}^{n}$ are define as follows

$$
\mathbf{W}_{i j}^{n}=\exp -\frac{\left\|\mathbf{c}_{i}-\mathbf{c}_{j}\right\|^{2}}{\sigma}
$$

where $\mathbf{c}_{i}$ and $\mathbf{c}_{j}$ are the mean spectral responses of homogeneous regions $G_{i}$ and $G_{j}$, respectively. By simplifying Equation (16), we get

$$
\begin{aligned}
\frac{1}{2} \sum_{i, j=1}^{Q}\left\|\alpha_{i}-\alpha_{j}\right\| \mathbf{W}_{i j}^{n} & =\sum_{i=1}^{Q} \alpha_{i}^{T} \alpha_{i} \mathbf{D}_{i i}-\sum_{i, j=1}^{Q} \alpha_{i}^{T} \alpha_{j} \mathbf{W}_{i j}^{n} \\
& =\operatorname{Tr}\left(\mathbf{R D}^{n} \mathbf{R}^{T}\right)-\operatorname{Tr}\left(\mathbf{R} \mathbf{W}^{n} \mathbf{R}^{T}\right) \\
& =\operatorname{Tr}\left(\mathbf{R} \mathbf{L}^{n} \mathbf{R}^{T}\right)
\end{aligned}
$$

where $\operatorname{Tr}()$ is the trace of the matrix, $\mathbf{L}^{n}$ and $\mathbf{D}^{n}$ are the Laplacian matrix and the diagonal matrix defined as follows

$$
\begin{gathered}
\mathbf{L}^{n}=\mathbf{D}^{n}-\mathbf{W}^{n} \\
\mathbf{D}_{i i}^{n}=\sum_{j} \mathbf{W}_{i j}^{n}
\end{gathered}
$$

The distant repulsion is defined as follows

$$
\min _{\alpha} \frac{1}{2} \sum_{i=1}^{Q} \sum_{j=1}^{Q} \mathbf{W}_{i j}^{f} \exp \left(-\left\|\alpha_{i}-\alpha_{j}\right\|^{2}\right)
$$

where $\mathbf{W}_{i j}^{f}$ is calculated by

$$
\mathbf{W}_{i j}^{f}=\left\|\mathbf{c}_{i}-\mathbf{c}_{j}\right\|^{2}
$$

where $\mathbf{c}_{j}$ is the mean spectral response of homogeneous region that is among the $k$ farthest neighbors of region correspond to $\mathbf{c}_{i}$. Equation (21) tells that if $\mathbf{c}_{i}$ and $\mathbf{c}_{j}$ are different from each other, this property will be preserved in $\alpha_{i}$ and $\alpha_{j}$.

Combining Equations (18) and (21), $\alpha_{i}$ can be obtained by minimizing following equation

$$
\begin{aligned}
f(\mathbf{M}, \mathbf{A})= & \frac{1}{2}\left\|\mathbf{c}_{q}-\mathbf{M} \alpha_{q}\right\|_{F}^{2} \\
& +\sum_{q=1}^{Q} \lambda_{q}\left\|\alpha_{q}\right\|_{1 / 2}+\frac{\gamma_{1}}{2} \operatorname{Tr}\left(\mathbf{A} \mathbf{L}^{n} \mathbf{A}^{T}\right) \\
& +\frac{\gamma_{2}}{4} \sum_{i j}^{Q} \mathbf{W}_{i j}^{f} \exp \left(-\left\|\alpha_{i}-\alpha_{j}\right\|^{2}\right)
\end{aligned}
$$

where the first and the second terms form the sparsity regularized NMF model, and the third and the fourth terms are the structure preservation terms.

To optimize the function, we define

$$
\begin{gathered}
\mathbf{C}=\left[\mathbf{c}_{1}, \mathbf{c}_{2}, \ldots, \mathbf{c}_{Q}\right] \\
\mathbf{A}=\left[\alpha_{1} \alpha_{2}, \ldots, \alpha_{Q}\right] \\
\Lambda=\left(\begin{array}{cccc}
\lambda_{1} & 0 & \ldots & 0 \\
0 & \lambda_{2} & \ldots & 0 \\
0 & 0 & \ldots & 0 \\
0 & 0 & \ldots & \lambda_{Q}
\end{array}\right)
\end{gathered}
$$

Then the Equation (23) can be rewritten as follows:

$$
\begin{aligned}
f(\mathbf{M}, \mathbf{A})= & \frac{1}{2}\|\mathbf{C}-\mathbf{M} \mathbf{A}\|_{F}^{2} \\
& +\left\|\mathbf{A} \Lambda^{2}\right\|_{1 / 2}+\frac{\gamma_{1}}{2} \operatorname{Tr}\left(\mathbf{A L}^{n} \mathbf{A}^{T}\right) \\
& +\frac{\gamma_{2}}{4} \sum_{i j}^{n} \mathbf{W}_{i j}^{f} \exp \left(-\left\|\alpha_{i}-\alpha_{j}\right\|^{2}\right)
\end{aligned}
$$




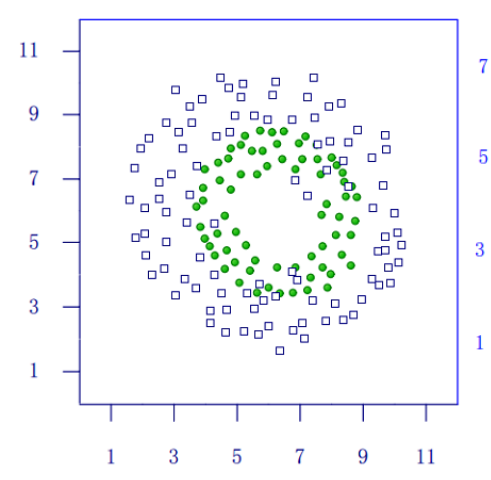

(a) Original data

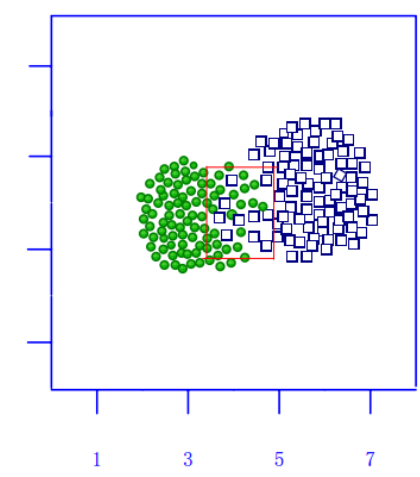

(b) Local affinity

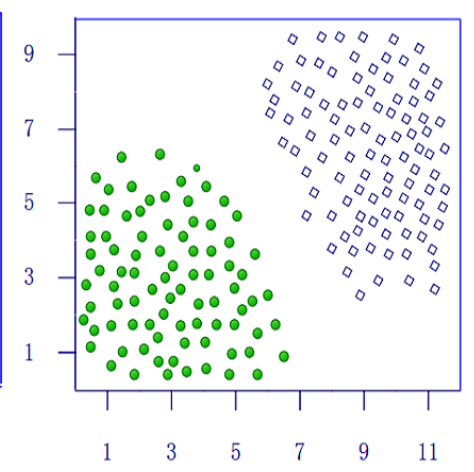

(c) Distant repulsion

Fig. 1. Illustration of structured information after abundance estimation.

We follow the method by Li et al [43] to solve Equation (27). Define a Lagrangian function as follows

$$
\begin{aligned}
\mathcal{L}= & \frac{1}{2}\|\mathbf{C}-\mathbf{M A}\|_{F}^{2} \\
& +\left\|\mathbf{A} \Lambda^{2}\right\|_{1 / 2}+\frac{\gamma_{1}}{2} \operatorname{Tr}\left(\mathbf{A L}^{n} \mathbf{A}^{T}\right) \\
& +\frac{\gamma_{2}}{4} \sum_{i j}^{n} \mathbf{W}_{i j}^{f} \exp \left(-\left\|\alpha_{i}-\alpha_{j}\right\|^{2}\right) \\
& +\operatorname{Tr}\left(\Psi \mathbf{M}^{T}\right)+\operatorname{Tr}\left(\Phi \mathbf{A}^{T}\right)
\end{aligned}
$$

Taking the derivative of abundance matrix $\mathbf{A}$, we get

$$
\frac{\partial \mathcal{L}}{\partial \mathbf{A}}=-\mathbf{M}^{T} \mathbf{C}+\mathbf{M}^{T} \mathbf{M} \mathbf{A}+\gamma_{1} \mathbf{A} \mathbf{L}+\frac{1}{2} \mathbf{A}^{-\frac{1}{2}} \Lambda+\Phi
$$

where

$$
\begin{gathered}
\mathbf{L}=\mathbf{L}^{n}-\eta \tilde{\mathbf{L}}^{f} \\
\eta=\gamma_{2} / \gamma_{1}
\end{gathered}
$$

$\mathbf{L}^{n}$ matrix is the Laplacian matrix of the k-nearest neighbours. $\tilde{\mathbf{L}}^{f}$ is defined as follows

$$
\tilde{\mathbf{L}}^{f}=\tilde{\mathbf{D}}^{f}-\tilde{\mathbf{W}}^{f}
$$

where

$$
\begin{gathered}
\tilde{\mathbf{W}}_{i j}^{f}=\mathbf{W}_{i j}^{f} \exp \left(-\left\|\alpha_{i}-\alpha_{j}\right\|^{2}\right) \\
\tilde{\mathbf{D}}_{i i}^{f}=\sum_{j} \tilde{\mathbf{W}}_{i j}^{r}
\end{gathered}
$$

Using the Karush-Kuhn-Tucker (KKT) conditions $\Phi_{p q} \mathbf{A}_{p q}=$ 0 . We get the following equation

$$
\left(-\mathbf{M}^{T} \mathbf{C}+\mathbf{M}^{T} \mathbf{M} \mathbf{A}+\frac{1}{2} \mathbf{A}^{-\frac{1}{2}} \Lambda+\gamma_{1} \mathbf{A L}\right)_{p q} \mathbf{A}_{p q}=0
$$

Following the method by Li et al [43], let $\mathbf{L}=\mathbf{L}^{+}-\mathbf{L}^{-}$, where $\mathbf{L}_{i j}^{+}=\left(\left|\mathbf{L}_{i j}\right|+\mathbf{L}_{i j}\right) / 2$ and $\mathbf{L}_{i j}^{-}=\left(\left|\mathbf{L}_{i j}\right|-\mathbf{L}_{i j}\right) / 2, \mathbf{A}_{p q}$ can be updated as follows

$$
\mathbf{A}_{p q} \leftarrow \mathbf{A}_{p q} \cdot * \frac{\left(\mathbf{M}^{T} \mathbf{C}+\gamma_{1} \mathbf{A L}^{-}\right)_{p q}}{\left(\mathbf{M}^{T} \mathbf{M} \mathbf{A}+\frac{1}{2} \mathbf{A}^{-\frac{1}{2}} \Lambda+\gamma_{1} \mathbf{A} \mathbf{L}^{+}\right)_{p q}}
$$

\section{Implementation Issues}

In all NMF based methods, we initialize endmember matrix $\mathbf{M}$ by the VCA method [11] and then calculate matrix $\mathbf{R}$ by the FCLS method [44]. On synthetic data, since the number of endmembers are known when generating dataset, we simply used the same number as the endmember number in our model. On real world data, we set the number of endmembers as a fixed value following several studies on the real world datasets [45]. Although the number of endmembers is an important parameter, how it can be optimally determined is beyond the scope of our paper. Relevant solutions can be found, for example, in [46], [47].

There are two stopping criteria in our implementation. The first one is the maximum iteration number which is set to 3000 . The second one is the gradient difference of the objective function between the current and the previous iterations:

$$
\left|\operatorname{obj}\left(\mathbf{M}^{i}, \mathbf{R}^{i}\right)\right| \leq \epsilon\left|\operatorname{obj}\left(\mathbf{M}^{i-1}, \mathbf{R}^{i-1}\right)\right|
$$

where $\epsilon$ is set to $10^{-4}$ in the experiments. Once either of these criteria is met, the optimisation process terminates.

The procedure of our homogeneous region regularized NMF method is described below

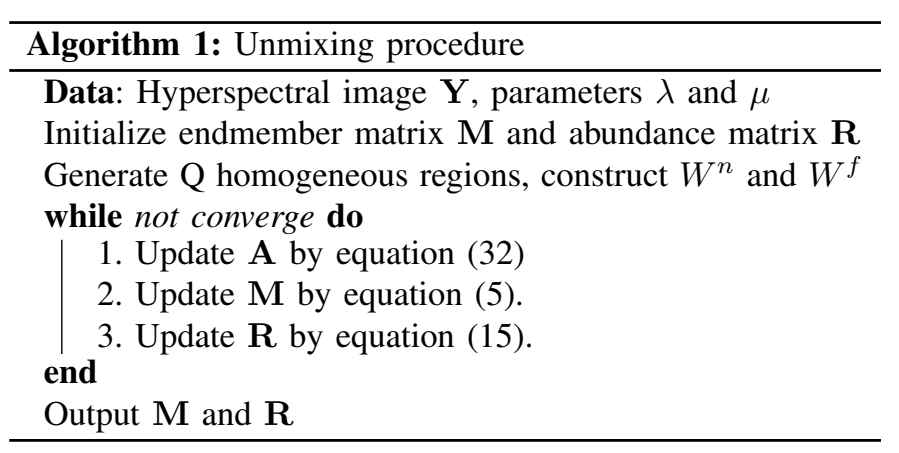

\section{EXPERIMENTS}

A series of experiments were developed to evaluate the performance of the proposed region based NMF (R-NMF) method. We also compared it against several baseline unmixing methods, including VCA [11], super pixel based VCA (S-VCA) [35], $L_{1 / 2}$-NMF [25], manifold regularized 
NMF (MRS-NMF) [29] and Double constrained NMF (DNMF) [30].

Two evaluation criteria are used: spectral angle distance (SAD) and root mean squared error (RMSE). The SAD is used to compare the similarity of the $p$-th endmember signature $\mathbf{M}_{p}$ and its estimation $\widehat{\mathbf{M}}_{p}$, which is defined as

$$
\operatorname{SAD}_{p}=\arccos \left(\frac{\mathbf{M}_{p}^{T} \widehat{\mathbf{M}}_{p}}{\left\|\mathbf{M}_{p}\right\|\left\|\widehat{\mathbf{M}}_{p}\right\|}\right)
$$

The RMSE is used to evaluate the the abundance estimation, which is defined as

$$
\operatorname{RMSE}_{p}=\left(\frac{1}{N}\left|\mathbf{R}_{p}-\widehat{\mathbf{R}}_{p}\right|^{2}\right)^{\frac{1}{2}}
$$

where $\widehat{\mathbf{R}}_{p}$ is the reference abundance matrix for the $p^{\text {th }}$ endmember.

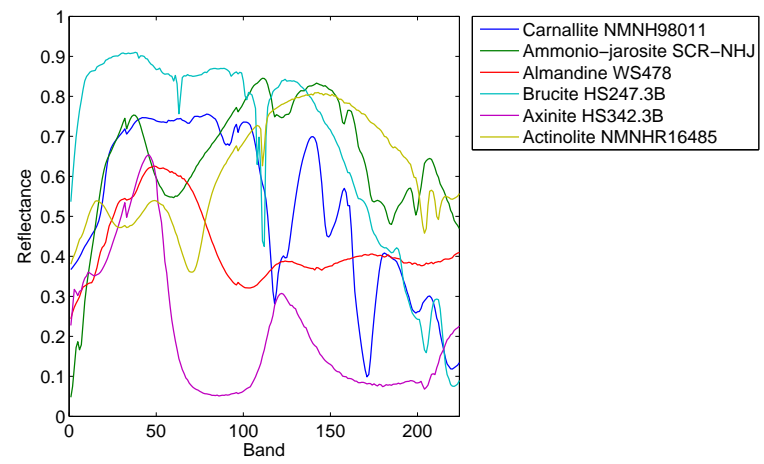

Fig. 2. Example endmember spectra used in synthetic data.

\section{A. Synthetic Data}

We first evaluate and analyze the proposed method on synthetic data. Six spectral signatures were chosen from the USGS digital spectral library [48] to generate the synthetic data, which are shown in Fig. 2. Similar to [49], there were 5 steps to generate this synthetic data.

1) Generate an image which contains $z^{2} \times z^{2}$ pixels and divide it into $z \times z$ regions.

2) In each region the same type of ground cover is initialized with one of the endmembers chosen randomly.

3) $\mathrm{A}(z+1) \times(z+1)$ low pass filter is applied to the image to generate mixed data.

4) For each pixel whose abundance is larger than 0.8 , the abundance at the pixel is replaced with a mixture of all endmembers with equal abundances.

5) Zero-mean Gaussian noise is added to the synthetic data. The signal to noise ratio(SNR) is defined as

$$
S N R=10 \log _{10} \frac{E\left[\mathbf{y}^{T} \mathbf{y}\right]}{E\left[\mathbf{e}^{T} \mathbf{e}\right]}
$$

where $y$ and $e$ are the observation and noise at a pixel. $E[\cdot]$ denotes the expectation operator.

Fig. 3(a) displays the synthetic data of band 100 with SNR of $25 \mathrm{~dB}$ and Figs. 3(b)-(f) show the abundance maps of five endmembers.

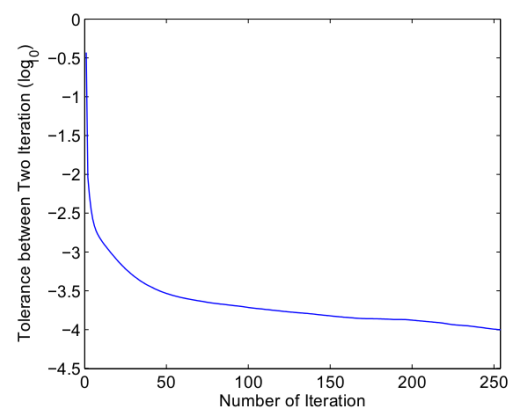

Fig. 4. Convergence curve of R-NMF.

1) Experiment 1 (Convergence Analysis): It is difficult to prove the convergence of the proposed algorithm. We did it in an alternative way by plotting the convergence curve of the proposed algorithm in Fig. 4. It can be seen that at the beginning, tolerance of the object function is quite large. The error drops quickly with the optimization iterations, and changes slowly when the iteration number passes 100 .

2) Experiment 2 (Parameters): In our method, the bias $\rho$ and the minimum region size $T$ in section III-A are important parameters for homogeneous region segmentation. Fig. 5 displays the segmentation results with different $T$ from 10 to 200 . It can be seen that when the size $T$ is small, the segmentation results is very trivial, but when $T$ is too large, different materials may be assigned to the same region. Therefore, we set $T=50$ in the experiment. We also display the segmentation results with different $\sigma$ values in Fig. 6 . The figure shows that this parameter has less impact to the segmentation results, so we set $\sigma=0.01$.

$\mu$ in Equation (10) is a parameter that controls the similarity between estimated abundance and the abundance in homogeneous regions. To evaluate $\mu$, we calculated $\lambda_{q}$ using Equation (11) and changed $\mu$ from 0.1 to 1 . From Fig. 7, we can see that for SAD criterion the optimal value of $\mu$ is 0.3 while for RMSE it is 0.4 .We set $\mu$ as 0.3 in our experiments.

$\gamma_{1}$ and $\gamma_{2}$ are parameters that control the influence of the constraints on consistency of same material and diversity of different materials in the same homogeneous region. We changed $\gamma_{1}$ and $\gamma_{2}$ from 0.05 to 1 with an interval of 0.05 . Fig. 8 shows that the performance is better when $\gamma_{1}=0.1$ and $\gamma_{2}=0.15$. Therefore, we set $\gamma_{1}=0.1$ and $\gamma_{2}=0.15$ in our experiments.

3) Experiment 3 (Comparison of Different Methods): In this experiment, we compare the proposed R-NMF method with several baseline methods. We set $S N R=25$ and the total number of endmembers $P=6$. The code for each method was run for 10 times and then the means and standard deviations of SAD and RMSE were calculated. Fig. 9 shows the experimental results. The bars and error lines stand for the mean SAD and RMSE and their standard deviations, respectively. From the figure, we can see that the performance of our proposed method is better than its counterparts.

From Fig. 9 we can see that the performance of SAD and RMSE of all NMF based methods are better than those from VCA. This verifies that NMF based method is effective 


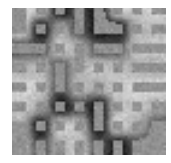

(a)

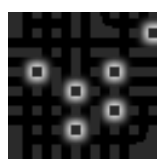

(b)

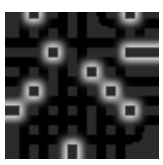

(c)

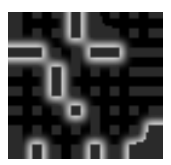

(d)

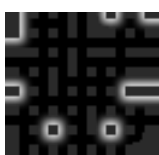

(e)

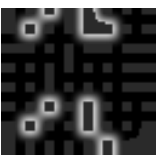

(f)

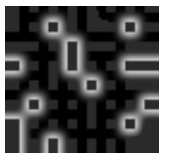

(g)

Fig. 3. Synthetic data. (a) Synthetic data of band 100. (b)-(g) Abundance maps.

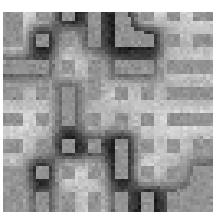

(a)

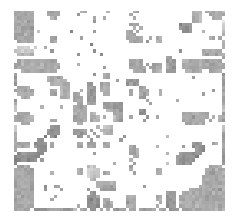

(b)

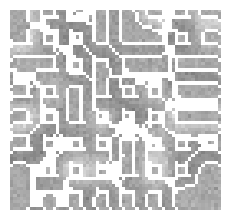

(c)

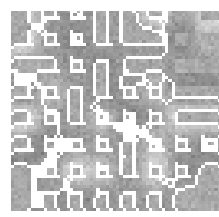

(d)

Fig. 5. Segmentation results. (a) Synthetic data of band 100. (b) Segmentation of band 100 with $T=10$. (c) Segmentation of band 100 with $T=50$. (e) Segmentation of band 100 with $T=200$.

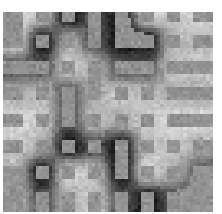

(a)

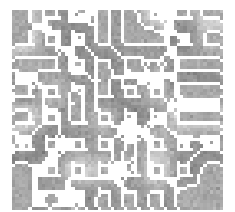

(b)

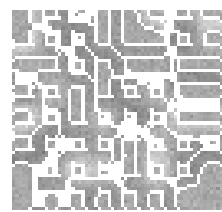

(c)

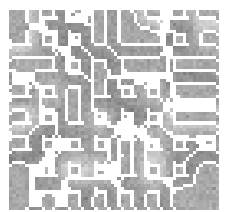

(d)

Fig. 6. Segmentation results. (a) Synthetic data of band 100. (b) Segmentation of band 100 with $\sigma=0.1$. (c) Segmentation of band 100 with $\sigma=0.01$. (e) Segmentation of band 100 with $\sigma=0.001$.

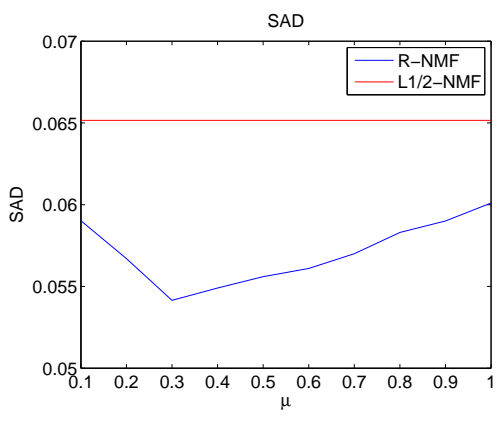

(a)

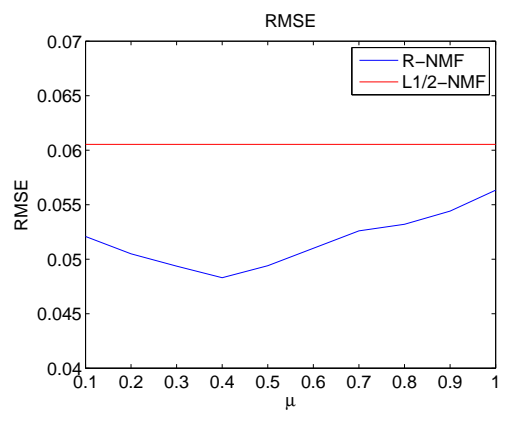

(b)

Fig. 7. Results under different values of $\mu$. (a) Mean SAD. (b) Mean RMSE.

when initialized with VCA-FCLS method. The performance of MRS-NMF is marginally better than $L_{1 / 2}$-NMF because it includes the manifold information between pixels in hyperspectral data and abundance matrix. The result of D-NMF is better than MRS-NMF because it has a clustering process which could maintain the structural information during the unmixing. For the proposed R-NMF method, the performance is better than all other methods. This is because we set consistency constraint in homogeneous regions and preserve the structural information between regions. Fig. 10 shows the comparison of the estimated spectral signatures by R-NMF (dashed lines) and the reference signatures extracted from the library (solid lines).

4) Experiment 4 (Robustness to Noise): In this experiment, different levels of SNR were applied to the synthetic data to verify the robustness of our method. We set SNR as $15 \mathrm{~dB}, 25 \mathrm{~dB}, 35 \mathrm{~dB}, 45 \mathrm{~dB}$ and infinity (noise free), respectively. Fig. 11 shows the SAD and RMSE results from different methods. It can be seen that when the value of SNR becomes large, the mean values of both SAD and RMSE become small. Therefore, noises have strong influences on the unmixing results. Fig. 11(a) shows that our method significantly outperforms all other methods on endmember estimation. Meanwhile, Fig. 11(b) also shows that the RMSE of our method is better than others no matter how the noise level changes. Our algorithm clearly is more robust to the influence of noise. 


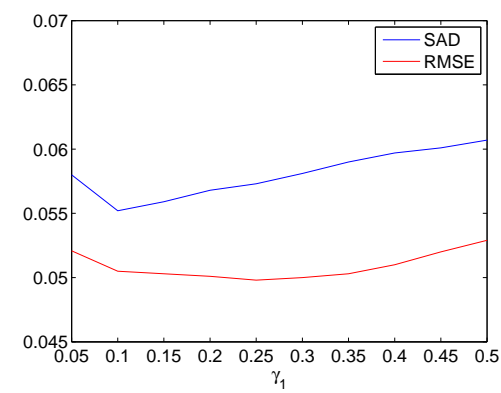

(a)

Fig. 8. Results under different values of $\gamma_{1}$ and $\gamma_{2}$. (a) $\gamma_{1}$. (b) $\gamma_{2}$.

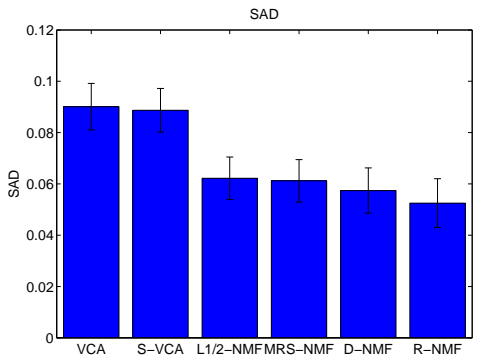

(a)

Fig. 9. Performance of different algorithms. (a) Mean SAD. (b) Mean RMSE.

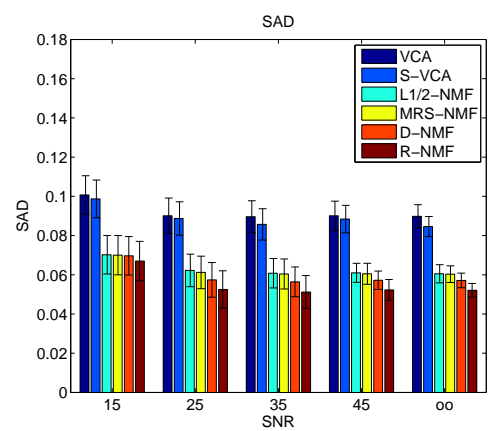

(a)

Fig. 11. The results of different SNRs. (a) Mean SAD; (b) Mean RMSE.

\section{B. Remote Sensing Data}

Three real-world datasets were used to evaluate the proposed R-NMF method: Jasper, Urban and Washington DC data sets. There are different numbers of endmembers in each dataset. We got the reference ground truth of endmembers following the method of Qian et al [50]. To match the reference spectra with the estimated spectra, we calculated the correlation between the spectral responses and then assign the estimated endmember to the nearest reference endmember. Note that there is no ground truth on the abundance of each endmember for these data, so it is difficult to give quantitative evaluation on the abundance. Therefore we only display the abundance map of each endmember.

1) Jasper Ridge Dataset: The Jasper Ridge dataset contains 224 bands covering the wavelengths from $380 \mathrm{~nm}$ to $2500 \mathrm{~nm}$,

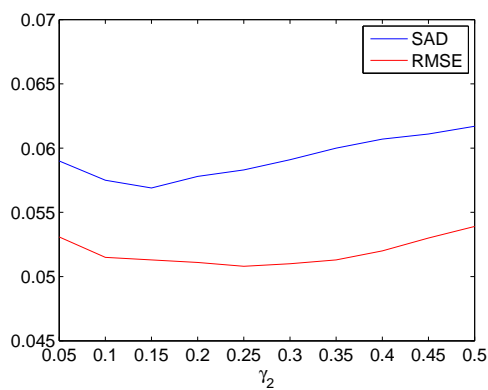

(b)

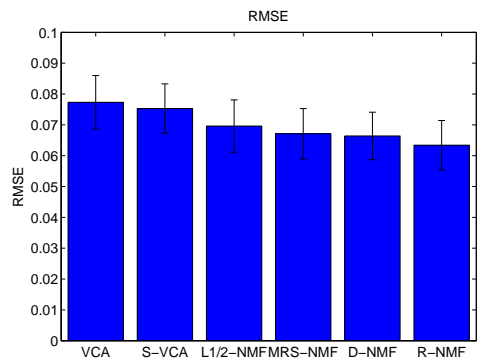

(b)

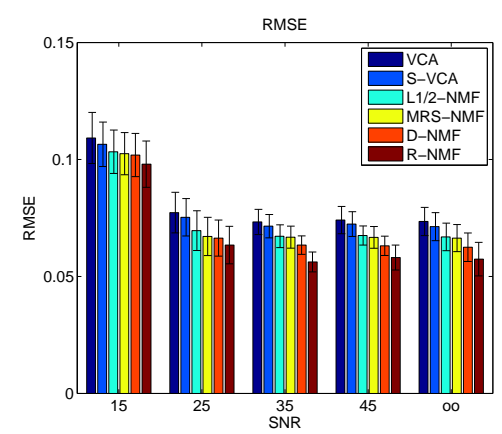

(b)

with a $10 \mathrm{~nm}$ spectral resolution. The size of a single band image is $512 \times 614$. In the experiments, we crop the original image to a subimage of $100 \times 100$ pixels from location (105, 269) [45]. Fig. 12 displays the 50th, 90th, and 130th bands of the subimage that has been used in the experiments. We removed the low SNR and water-vapor absorption bands (1-3, 108-112, 154-166 and 220-224), which yielded 198 bands out of the original 224. 4 types of endmembers were analyzed in the experiments, i.e. soil, water, tree and road.

In the experiment, we calculated the mean SAD values and the variances of these 4 endmembers. The results in Table I show that the proposed method has significantly outperformed VCA, S-VCA, $L_{1 / 2}$-NMF, MRS-NMF, and DNMF methods. There are large homogeneous regions in this data set, our method better uses the spatial information of these 
TABLE I

RESULTS OF DIFFERENT METHODS ON JASPER DATASET.

\begin{tabular}{ccccccc}
\hline \hline Method & VCA & S-VCA & $L_{1 / 2}$-NMF & MRS-NMF & D-NMF & R-NMF \\
\hline Tree & $0.1320 \pm 0.0297$ & $0.1332 \pm 0.0205$ & $0.0494 \pm 0.0030$ & $0.0546 \pm 0.0028$ & $0.0501 \pm 0.0025$ & $\mathbf{0 . 0 2 9 9} \pm \mathbf{0 . 0 0 3 1}$ \\
Water & $0.1822 \pm 0.0357$ & $0.1592 \pm 0.0253$ & $0.1680 \pm 0.0090$ & $0.1716 \pm 0.0089$ & $0.1707 \pm 0.0088$ & $\mathbf{0 . 1 5 5 0} \pm \mathbf{0 . 0 0 8 5}$ \\
Soil & $0.1967 \pm 0.0534$ & $0.0699 \pm 0.0367$ & $0.0725 \pm 0.0019$ & $0.0672 \pm 0.0019$ & $0.0606 \pm 0.0018$ & $\mathbf{0 . 0 4 2 7} \pm \mathbf{0 . 0 0 2 2}$ \\
road & $0.0901 \pm 0.0008$ & $0.0935 \pm 0.0010$ & $0.0565 \pm 0.0027$ & $0.0499 \pm 0.0021$ & $0.0437 \pm 0.0020$ & $\mathbf{0 . 0 3 8 6} \pm \mathbf{0 . 0 0 2 3}$ \\
\hline Mean & $0.1503 \pm 0.0314$ & $0.1136 \pm 0.0209$ & $0.0866 \pm 0.0041$ & $0.0858 \pm 0.0039$ & $0.0813 \pm 0.0038$ & $\mathbf{0 . 0 6 6 5} \pm \mathbf{0 . 0 0 4 0}$ \\
\hline \hline
\end{tabular}
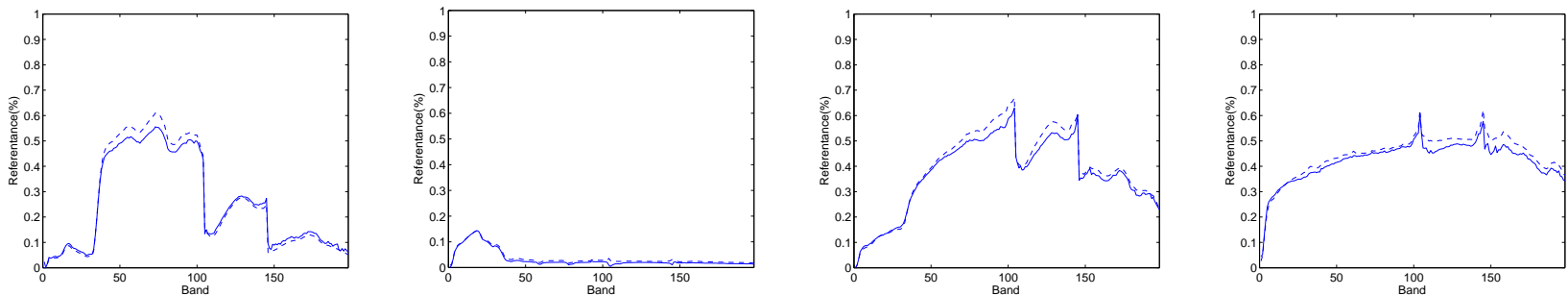

Fig. 13. Endmembers of Jasper estimated by R-NMF. Solid lines denote the reference endmembers and dashed lines denote the estimated endmembers. (a) Tree. (b) Water. (c) Soil. (d) Road.

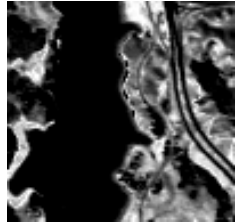

(a)

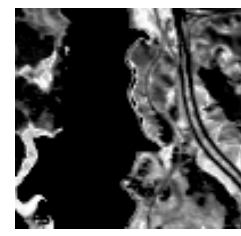

(e)

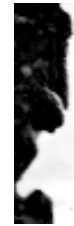

(b)

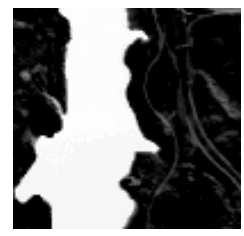

(f)

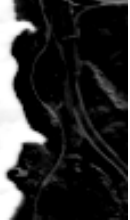

b)

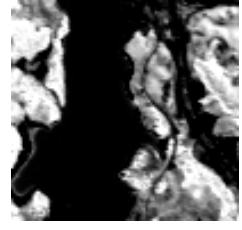

(c)

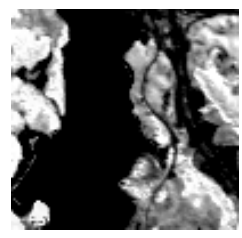

(g)

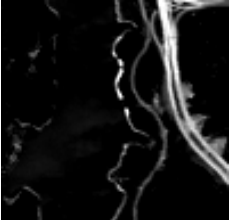

(d)

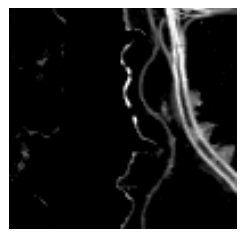

(h)

Fig. 14. Abundance map of Jasper data set.(a)-(d) Reference abundance map of each endmember: (a) Soil; (b) Water; (c) Tree; (d) Road. (e)-(h) Estimated abundance map of each endmember: (e) Soil; (f) Water; (g) Tree; (h) Road.

homogeneous region. Fig. 13 shows the estimated endmember signatures with the references. Fig. 14 shows the reference abundance maps and estimated abundance maps respectively. From these figures, it can be seen that our method produced abundance map and endmember signatures very close to the references.

2) Urban HYDICE Dataset: We also ran experiments on the widely used Urban image. This image was obtained by the HYDICE sensor [25]. The image depicts the scene displayed in Fig. 17 and is of size $307 \times 307$. This image is composed of 210 spectral channels with spectral resolution of $10 \mathrm{~nm}$ acquired in the $400 \mathrm{~nm}$ to $2500 \mathrm{~nm}$ region. After low SNR bands had been removed (channels 1-4, 76, 87, 101-111, 136-153, and 198-210), 162 bands remained for the experiments, i.e., $H=162$.

In the experiments, we set the number of endmembers as 4, including road, grass, asphalt and tree. Table II shows the mean SAD of endmember estimations with different methods. From this table, we can see that the performance of our proposed RNMF is better than other methods. Fig. 18 shows the estimated endmember signatures and their references. It can be seen that the output of R-NMF method is very close to the references. Fig. 19(a)-(d) show the abundance maps of four endmembers compare to the reference abundance map in Fig. 19(e)-(h). The results are quite reasonable comparing with references.

3) Washington DC Mall Dataset: The last experiments were conducted on Washington DC Mall dataset captured by Urban Hyperspectral Digital Imagery Collection Experiment (HYDICE) sensor. Due to the large size, we crop the original image to a sub-image with size of $150 \times 150$. After low-SNR bands are removed (channels 103-106, 138-148, 207-210), 191 bands remain. The sub-image is displayed in Fig. 20. Following Jia and Qian [27], we set five endmembers in the image: tree, grass, water, roof, and street.

In the experiment, we calculated the mean SAD values and their variances of 5 endmembers. The results in Table III show that the proposed method R-NMF has outperformed VCA, $L_{1 / 2}$-NMF, MRS-GNMF and D-NMF methods. Fig. 16(a)-(e) show the abundance maps of five endmembers compared to the reference abundance map in Fig. 16(f)-(j). Fig. 15 shows 

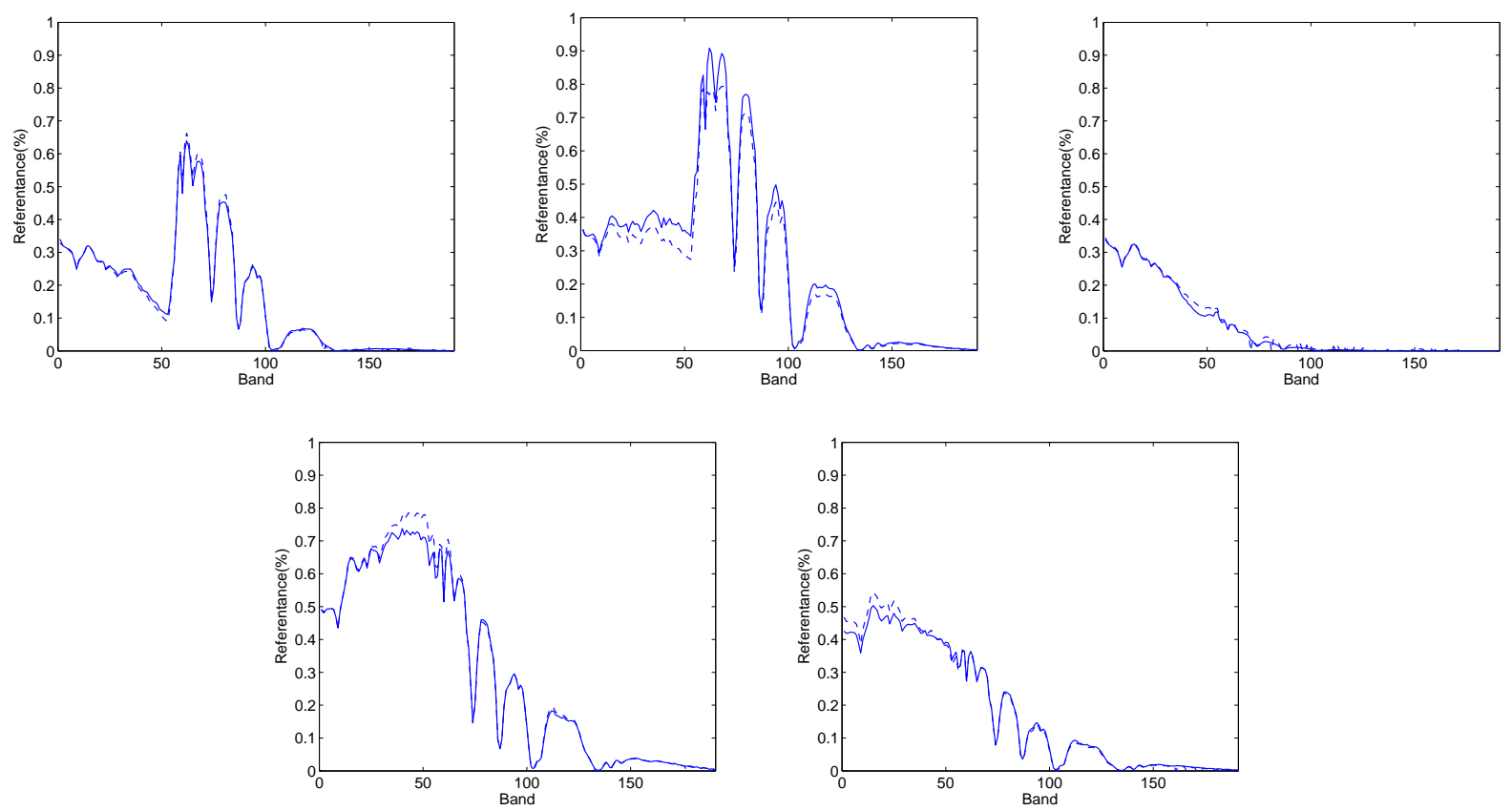

Fig. 15. Endmembers of Washington DC estimated by R-NMF. Solid lines denote the reference endmembers and dashed lines denote the estimated endmembers. (a) Tree. (b) Grass. (c) Water. (d) Roof. (e) Street.

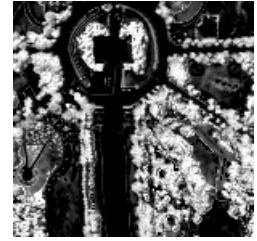

(a)

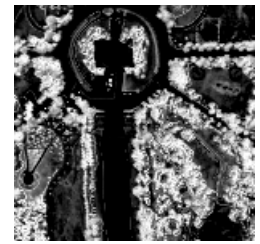

(f)

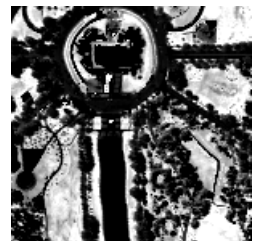

(b)

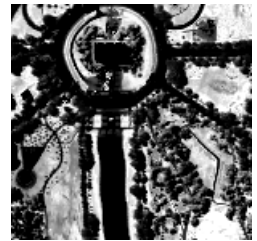

(g)

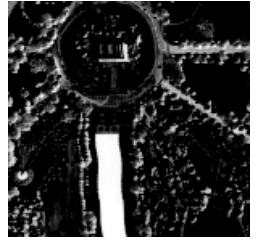

(c)

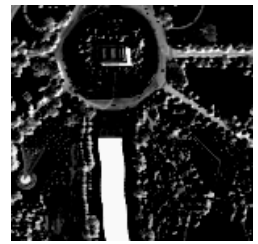

(h)

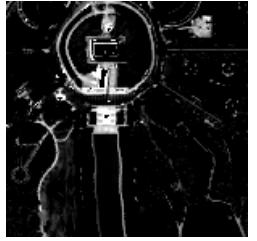

(d)

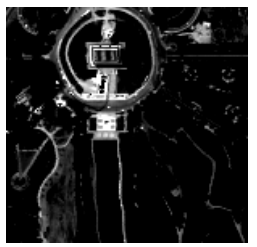

(i)

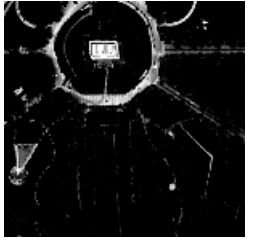

(e)

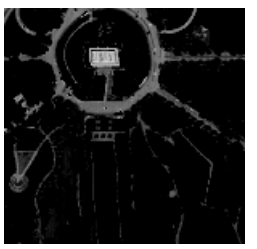

(j)

Fig. 16. Abundance map of Jasper data set. (a)-(e) Reference abundance map of each endmembers: (a) Tree; (b) Grass; (c) Water; (d) Roof; (e) Street. (f)-(j) Estimated abundance map of each endmembers: (f) Tree; (g) Grass; (h) Water; (i) Roof; (j) Street.

the estimated endmembers and the reference ones. The result is also close to the references.

\section{CONCLUSION}

In this paper, a region based structure preserving NMF (R-NMF) method has been introduced for hyperspectral unmixing. This method first divides hyperspectral image into homogeneous regions using a graph cut method. By imposing constraints on consistency and structure information in homogeneous regions, R-NMF preserves the similarity and dissimilarity of raw data in homogeneous regions after abundance estimation. A series of experiments on both synthetic and real data show that the proposed method has achieved better performance than several baseline methods including VCA, SVCA, $L_{1 / 2}$-NMF, MRS-NMF and D-NMF. In the future, we will develop an adaptive constraint which could process the unmixing problem according to different properties of different regions.

\section{REFERENCES}

[1] A. F. Goetz, G. Vane, J. E. Solomon, and B. N. Rock, "Imaging spectrometry for earth remote sensing," Science, vol. 228, no. 4704, pp. 1147-1153, 1985.

[2] M. Fauvel, J. A. Benediktsson, J. Chanussot, and J. R. Sveinsson, "Spectral and spatial classification of hyperspectral data using svms and morphological profiles," IEEE Transactions on Geoscience and Remote Sensing, vol. 46, no. 11, pp. 3804-3814, 2008.

[3] C. C. Lelong, P. C. Pinet, and H. Poilvé, "Hyperspectral imaging and stress mapping in agriculture: a case study on wheat in beauce (france)," Remote sensing of environment, vol. 66, no. 2, pp. 179-191, 1998. 
TABLE II

RESULTS OF DIFFERENT METHODS ON URBAN DATASET.

\begin{tabular}{ccccccc}
\hline \hline Method & VCA & S-VCA & $L_{1 / 2}$-NMF & MRS-NMF & D-NMF & R-NMF \\
\hline Road & $0.6091 \pm 0.0219$ & $0.5083 \pm 0.0213$ & $0.0368 \pm 0.0015$ & $0.0422 \pm 0.0014$ & $0.0403 \pm 0.0010$ & $\mathbf{0 . 0 3 6 6} \pm \mathbf{0 . 0 0 0 9}$ \\
Grass & $0.5501 \pm 0.0291$ & $0.4913 \pm 0.0271$ & $0.1908 \pm 0.0058$ & $0.1838 \pm 0.0060$ & $0.1374 \pm 0.0051$ & $\mathbf{0 . 1 3 1 6} \pm \mathbf{0 . 0 0 3 2}$ \\
Asphalt & $0.1661 \pm 0.0005$ & $\mathbf{0 . 1 6 3 1} \pm \mathbf{0 . 0 0 2 0}$ & $0.2829 \pm 0.0051$ & $0.2757 \pm 0.0078$ & $0.2896 \pm 0.0045$ & $0.2474 \pm 0.0089$ \\
Tree & $0.0980 \pm 0.0267$ & $0.0931 \pm 0.0231$ & $\mathbf{0 . 0 8 9 1} \pm \mathbf{0 . 0 0 4 5}$ & $0.0900 \pm 0.0044$ & $0.0946 \pm 0.0047$ & $0.0914 \pm 0.0053$ \\
\hline Mean & $0.3558 \pm 0.0195$ & $0.3140 \pm 0.0184$ & $0.1499 \pm 0.0042$ & $0.1479 \pm 0.0049$ & $0.1404 \pm 0.0038$ & $\mathbf{0 . 1 2 6 8} \pm \mathbf{0 . 0 0 4 5}$ \\
\hline \hline
\end{tabular}
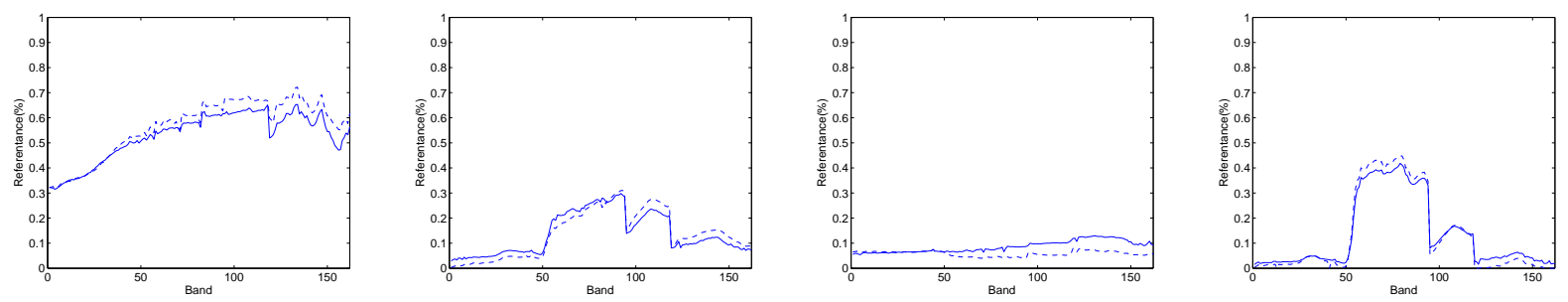

Fig. 18. Endmembers of HYDICE Urban estimated by R-NMF. Solid lines denote the reference endmembers and dashed lines denote the estimated endmembers. (a) Road. (b) Grass. (c) Asphalt. (d) Tree.

TABLE III

RESULTS OF DIFFERENT METHODS ON WASHINGTON DC DATASET.

\begin{tabular}{ccccccc}
\hline \hline Method & VCA & S-VCA & $L_{1 / 2}$-NMF & MRS-NMF & D-NMF & R-NMF \\
\hline Tree & $0.1988 \pm 0.0248$ & $0.1763 \pm 0.0213$ & $0.0588 \pm 0.0001$ & $0.0581 \pm 0.0022$ & $0.0596 \pm 0.0012$ & $\mathbf{0 . 0 1 9 9} \pm \mathbf{0 . 0 0 4 6}$ \\
Grass & $0.2109 \pm 0.0099$ & $0.2013 \pm 0.0093$ & $0.1667 \pm 0.0081$ & $\mathbf{0 . 1 6 6 5} \pm \mathbf{0 . 0 0 2 3}$ & $0.1727 \pm 0.0085$ & $0.1859 \pm 0.0088$ \\
Water & $0.3714 \pm 0.0348$ & $0.3215 \pm 0.0321$ & $0.0658 \pm 0.0032$ & $0.0633 \pm 0.0058$ & $0.0655 \pm 0.0032$ & $\mathbf{0 . 0 5 0 1} \pm \mathbf{0 . 0 0 3 7}$ \\
Roof & $0.1570 \pm 0.0236$ & $0.1473 \pm 0.0221$ & $0.0603 \pm 0.0014$ & $0.0601 \pm 0.0003$ & $0.0612 \pm 0.0019$ & $\mathbf{0 . 0 5 0 5} \pm \mathbf{0 . 0 0 2 5}$ \\
Street & $0.4326 \pm 0.0308$ & $0.3815 \pm 0.0296$ & $0.1094 \pm 0.0246$ & $0.1107 \pm 0.0094$ & $0.0943 \pm 0.0266$ & $\mathbf{0 . 0 7 9 0} \pm \mathbf{0 . 0 2 2 3}$ \\
\hline Mean & $0.2741 \pm 0.0248$ & $0.2456 \pm 0.0229$ & $0.0922 \pm 0.0074$ & $0.0917 \pm 0.0040$ & $0.0906 \pm 0.0083$ & $\mathbf{0 . 0 7 7 1} \pm \mathbf{0 . 0 0 8 3}$ \\
\hline \hline
\end{tabular}

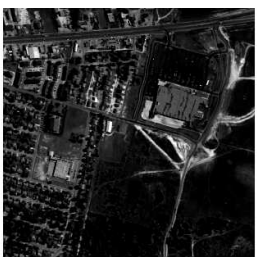

(a)

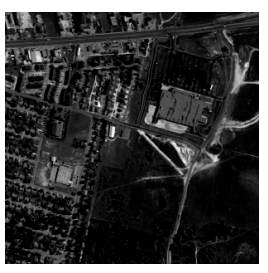

(e)

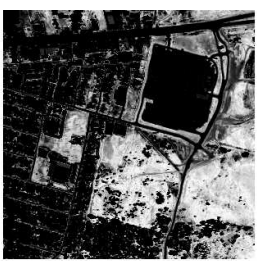

(b)

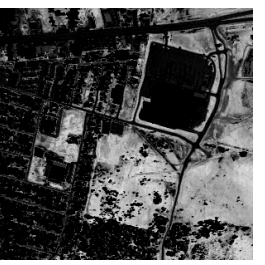

(f)

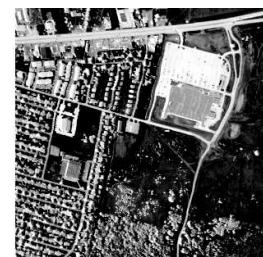

(c)

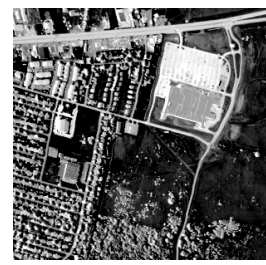

(g)

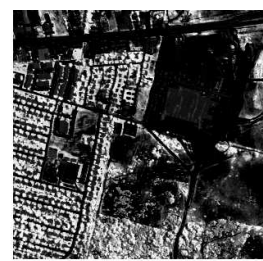

(d)

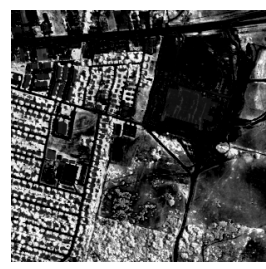

(h)

Fig. 19. Abundance map of Jasper data set. (a)-(d) Reference abundance map of each endmember: (a) Road; (b) Grass; (c) Asphalt; (d)Tree. (e)-(h) Estimated abundance map of each endmember: (e) Road; (f) Grass; (g) Asphalt; (h) Tree.

[4] R. Resmini, M. Kappus, W. Aldrich, J. Harsanyi, and M. Anderson, "Mineral mapping with hyperspectral digital imagery collection experiment (hydice) sensor data at cuprite, nevada, usa," International Journal of Remote Sensing, vol. 18, no. 7, pp. 1553-1570, 1997.

[5] N. Keshava and J. F. Mustard, "Spectral unmixing," IEEE Signal Processing Magazine, vol. 19, no. 1, pp. 44-57, 2002.

[6] A. Linear, "Foreword to the special issue on spectral unmixing of remotely sensed data," IEEE transactions on geoscience and remote sensing, vol. 49, no. 11, p. 4103, 2011.

[7] J. M. Bioucas-Dias, A. Plaza, N. Dobigeon, M. Parente, Q. Du, P. Gader, and J. Chanussot, "Hyperspectral unmixing overview: Geometrical, statistical, and sparse regression-based approaches," IEEE Journal of Selected Topics in Applied Earth Observations and Remote Sensing, vol. 5, no. 2, pp. 354-379, 2012.

[8] N. Keshava, "A survey of spectral unmixing algorithms," Lincoln Laboratory Journal, vol. 14, no. 1, pp. 55-78, 2003.

[9] M. E. Winter, "N-FINDR: an algorithm for fast autonomous spectral end-member determination in hyperspectral data," in SPIE International Symposium on Optical Science, Engineering, and Instrumentation. International Society for Optics and Photonics, 1999, pp. 266-275.

[10] J. W. Boardman, "Automating spectral unmixing of aviris data using convex geometry concepts," in JPL Airborne Geoscience Workshop Summaries 4th Annu., vol. 1. JPL Publication 93-26, 1993, pp. 11-14.

[11] J. M. Nascimento and J. M. Dias, "Vertex component analysis: A fast algorithm to unmix hyperspectral data," IEEE Transactions on Geoscience and Remote Sensing, vol. 43, no. 4, pp. 898-910, 2005. 


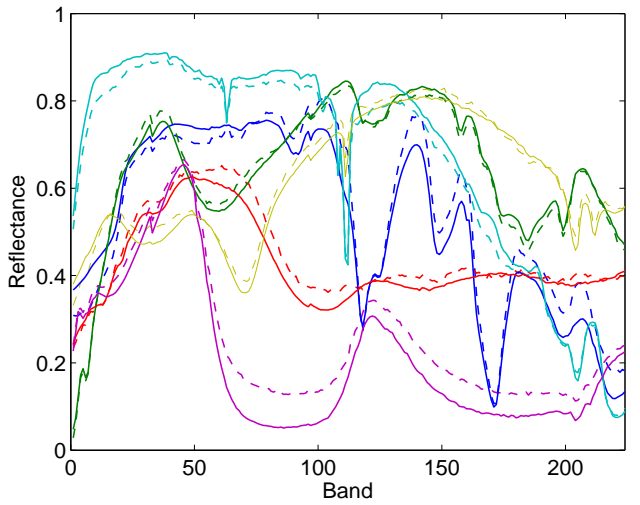

(a)

Fig. 10. Comparison of spectral signatures generated by R-NMF (dashed lines) and the reference signatures from the library (solid lines).
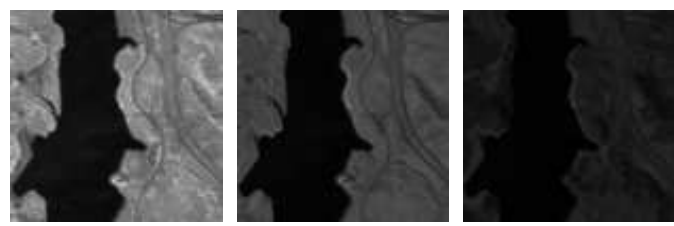

Fig. 12. Jasper dataset at bands 50, 90, 130.

[12] J. M. Bioucas-Dias, "A variable splitting augmented lagrangian approach to linear spectral unmixing," in First Workshop on Hyperspectral Image and Signal Processing: Evolution in Remote Sensing. IEEE, 2009, pp. $1-4$.

[13] M.-D. Iordache, J. Bioucas-Dias, and A. Plaza, "Sparse unmixing of hyperspectral data," IEEE Transactions on Geoscience and Remote Sensing, vol. 49, no. 6, pp. 2014-2039, June 2011.

[14] M. Iordache, J. Dias, and A. Plaza, "Collaborative sparse regression for hyperspectral unmixing," IEEE Transactions on Geoscience and Remote Sensing, vol. 52, no. 1, pp. 341-354, January 2013.

[15] N. Akhtar, F. Sahfait, and A. Mian, "Repeated constrained sparse coding with partial dictionaries for hyperspectral unmixing," in IEEE Winter Conference on Applications of Computer Vision (WACV), 2014, March 2014, pp. 953-960.

[16] M.-D. Iordache, J. Bioucas-Dias, and A. Plaza, "MUSIC-CSR: Hyperspectral unmixing via multiple signal classification and collaborative sparse regression," IEEE Transactions on Geoscience and Remote Sensing, vol. 52, no. 2, pp. 4364-4382, 2014.

[17] M.-D. Iordache, J. M. Bioucas-Dias, and A. Plaza, "Total variation spatial regularization for sparse hyperspectral unmixing," IEEE Transactions on Geoscience and Remote Sensing, vol. 50, no. 11, pp. 4484-4502, 2012.

[18] W. Tang, Z. Shi, Y. Wu, and C. Zhang, "Sparse unmixing of hyperspectral data using spectral a priori information," IEEE Transactions on Geoscience and Remote Sensing, vol. 53, no. 2, pp. 770-783, 2015.

[19] J. M. Nascimento and J. M. Bioucas Dias, "Does independent component analysis play a role in unmixing hyperspectral data?" IEEE Transactions on Geoscience and Remote Sensing, vol. 43, no. 1, pp. 175-187, 2005.

[20] M. Berman, H. Kiiveri, R. Lagerstrom, A. Ernst, R. Dunne, and J. F. Huntington, "Ice: A statistical approach to identifying endmembers in hyperspectral images," IEEE Transactions on Geoscience and Remote Sensing, vol. 42, no. 10, pp. 2085-2095, 2004.

[21] B. Zhang, L. Zhuang, L. Gao, W. Luo, Q. Ran, and Q. Du, "PSO-EM: A hyperspectral unmixing algorithm based on normal compositional model," IEEE Transactions on Geoscience and Remote Sensing, vol. 52, no. 12, pp. 7782-7792, 2014.

[22] V. P. Pauca, J. Piper, and R. J. Plemmons, "Nonnegative matrix factorization for spectral data analysis," Linear algebra and its applications, vol. 416, no. 1, pp. 29-47, 2006.

[23] N. Wang, B. Du, and L. Zhang, "An endmember dissimilarity con-
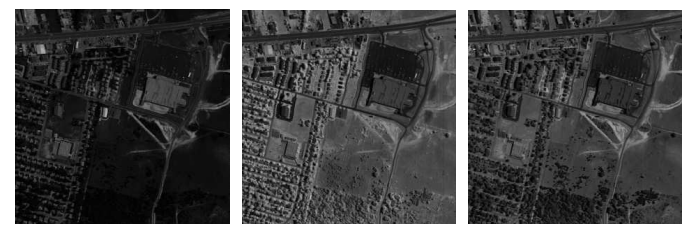

Fig. 17. Urban HYDICE dataset at bands 50, 80, 110.
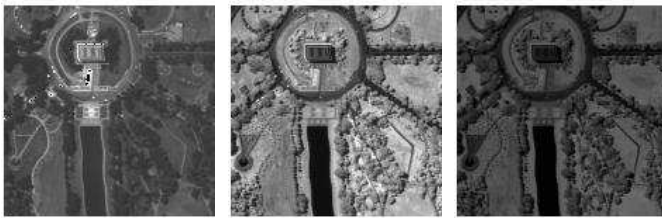

Fig. 20. Washington DC dataset at bands 30, 60, 90.

strained non-negative matrix factorization method for hyperspectral unmixing," IEEE Journal of Selected Topics in Applied Earth Observations and Remote Sensing, vol. 6, no. 2, pp. 554-569, April 2013.

[24] J. Liu, J. Zhang, Y. Gao, C. Zhang, and Z. Li, "Enhancing spectral unmixing by local neighborhood weights," IEEE Journal of Selected Topics in Applied Earth Observations and Remote Sensing, vol. 5, no. 5, pp. 1545-1552, 2012.

[25] Y. Qian, S. Jia, J. Zhou, and A. Robles-Kelly, "Hyperspectral unmixing via $L_{1 / 2}$ sparsity-constrained nonnegative matrix factorization," IEEE Transactions on Geoscience and Remote Sensing, vol. 49, no. 11, pp. 4282-4297, 2011.

[26] Z. Wu, S. Ye, J. Liu, L. Sun, and Z. Wei, "Sparse non-negative matrix factorization on GPUs for hyperspectral unmixing," IEEE Journal of Selected Topics in Applied Earth Observations and Remote Sensing, vol. 7, no. 8, pp. 3640-3649, 2014.

[27] S. Jia and Y. Qian, "Constrained nonnegative matrix factorization for hyperspectral unmixing," IEEE Transactions on Geoscience and Remote Sensing, vol. 47, no. 1, pp. 161-173, 2009.

[28] X. Liu, W. Xia, B. Wang, and L. Zhang, "An approach based on constrained nonnegative matrix factorization to unmix hyperspectral data," IEEE Transactions on Geoscience and Remote Sensing, vol. 49, no. 2, pp. 757-772, 2011

[29] X. Lu, H. Wu, Y. Yuan, P. Yan, and X. Li, "Manifold regularized sparse NMF for hyperspectral unmixing," IEEE Transactions on Geoscience and Remote Sensing, vol. 51, no. 5, pp. 2815-2826, 2013.

[30] X. Lu, H. Wu, and Y. Yuan, "Double constrained nmf for hyperspectral unmixing," IEEE Transactions on Geoscience and Remote Sensing, vol. 52, no. 5, pp. 2746-2758, 2014.

[31] Y. Yuan, Y. Feng, and X. Lu, "Projection-based NMF for hyperspectral unmixing," IEEE Journal of Selected Topics in Applied Earth Observations and Remote Sensing, vol. 8, no. 6, pp. 2632-2643, 2015.

[32] J. Li, J. M. Bioucas-Dias, A. Plaza, and L. Liu, "Robust collaborative nonnegative matrix factorization for hyperspectral unmixing," IEEE Transactions on Geoscience and Remote Sensing, vol. 54, no. 10, pp. 6076-6090, 2016.

[33] W. Tang, Z. Shi, and Z. An, "Nonnegative matrix factorization for hyperspectral unmixing using prior knowledge of spectral signatures," Optical Engineering, vol. 51, no. 8, pp. 087 001-1, 2012.

[34] L. Tong, J. Zhou, Y. Qian, X. Bai, and Y. Gao, "Nonnegative matrix factorization based hyperspectral unmixing with partially known endmembers," IEEE Transactions on Geoscience and Remote Sensing, vol. PP, no. 99, pp. 1-14, 2016.

[35] D. R. Thompson, L. Mandrake, M. S. Gilmore, and R. Castaño, "Superpixel endmember detection," IEEE Transactions on Geoscience and Remote Sensing, vol. 48, no. 11, pp. 4023-4033, 2010.

[36] G. Martín and A. Plaza, "Region-based spatial preprocessing for endmember extraction and spectral unmixing," IEEE Geoscience and Remote Sensing Letters, vol. 8, no. 4, pp. 745-749, 2011.

[37] O. Eches, N. Dobigeon, and J.-Y. Tourneret, "Enhancing hyperspectral image unmixing with spatial correlations," Geoscience and Remote Sensing, IEEE Transactions on, vol. 49, no. 11, pp. 4239-4247, 2011.

[38] D. D. Lee and H. S. Seung, "Learning the parts of objects by nonnegative matrix factorization," Nature, vol. 401, no. 6755, pp. 788-791, 1999. 
[39] — - "Algorithms for non-negative matrix factorization," in Advances in neural information processing systems, 2000, pp. 556-562.

[40] P. F. Felzenszwalb and D. P. Huttenlocher, "Efficient graph-based image segmentation," International Journal of Computer Vision, vol. 59, no. 2, pp. 167-181, 2004.

[41] J. Nievergelt and K. H. Hinrichs, Algorithms \& data structures. vdf Hochschulverl. an der ETH, 1999.

[42] M. Belkin and P. Niyogi, "Laplacian eigenmaps and spectral techniques for embedding and clustering." in Advances in Neural Information Processing Systems, vol. 14, 2001, pp. 585-591.

[43] Z. Li, J. Liu, and H. Lu, "Structure preserving non-negative matrix factorization for dimensionality reduction," Computer Vision and Image Understanding, vol. 117, no. 9, pp. 1175-1189, 2013.

[44] D. C. Heinz et al., "Fully constrained least squares linear spectral mixture analysis method for material quantification in hyperspectral imagery," IEEE Transactions on Geoscience and Remote Sensing, vol. 39, no. 3, pp. 529-545, 2001.

[45] F. Zhu, Y. Wang, S. Xiang, B. Fan, and C. Pan, "Structured sparse method for hyperspectral unmixing," ISPRS Journal of Photogrammetry and Remote Sensing, vol. 88, pp. $101-118,2014$.

[46] J. M. Bioucas-Dias and J. M. Nascimento, "Hyperspectral subspace identification," IEEE Transactions on Geoscience and Remote Sensing, vol. 46, no. 8, pp. 2435-2445, 2008.

[47] C.-I. Chang and Q. Du, "Estimation of number of spectrally distinct signal sources in hyperspectral imagery," IEEE Transactions on geoscience and remote sensing, vol. 42, no. 3, pp. 608-619, 2004.

[48] R. Clark, G. Swayze, R. Wise, E. Livo, T. Hoefen, R. Kokaly, and S. Sutley, "USGS digital spectral library splib06a: U.S. Geological Survey, Digital Data Series 231.” USGS, 2007.

[49] L. Miao and H. Qi, "Endmember extraction from highly mixed data using minimum volume constrained nonnegative matrix factorization," IEEE Transactions on Geoscience and Remote Sensing, vol. 45, no. 3 , pp. 765-777, 2007.

[50] Y. Qian, M. Ye, and J. Zhou, "Hyperspectral image classification based on structured sparse logistic regression and three-dimensional wavelet texture features," IEEE Transactions on Geoscience and Remote Sensing, vol. 51, no. 4, pp. 2276-2291, April 2013. 